\title{
Nosocomial lower respiratory tract infections in surgical wards
}

\author{
W J M Kinnear, R G Finch, R Pilkington, J T Macfarlane
}

\begin{abstract}
A six month prospective study of nosocomial lower respiratory tract infection was conducted in four general surgery wards and one urological surgery ward. Thirty eight cases were identified, representing $1 \%$ of the surgical admissions to these wards. Twenty eight of the infections followed abdominal surgery. Six patients died, five of whom had pre-existing cardiorespiratory disease. Potential pathogens were isolated in 21 patients, the commonest organisms being Streptococcus pneumoniae and Haemophilus infuenzae.
\end{abstract}

Figures from North America suggest that hospital acquired (nosocomial) respiratory tract infections occur in $0.5-5 \%$ of patients in hospital, account for $10-15 \%$ of all hospital acquired infections, and rank third in frequency of infection, coming after urinary tract and wound infection. Respiratory tract infections account for the greatest number of deaths due to nosocomial infection, and estimates suggest , that 20000-100000 patients die from nosocomial pneumonia in North America. ${ }^{12}$ The incidence of nosocomial respiratory tract infections in the United Kingdom is not known. A six week prospective study performed in the acute medical, general surgical, and thoracic surgical wards of the City Hospital, Nottingham, in 1986 identified 16 patients with nosocomial pneumonia, of whom seven died. ${ }^{3}$

Recent studies in Nottingham and elsewhere have shown that an appreciable proportion of community acquired respiratory tract infections are caused by atypical and viral pathogens. ${ }^{45}$ No data on the aetiology of nosocomial respiratory tract infections in the United Kingdom have been published since the report of a small study from Edinburgh in $1984 .^{6}$ Studies from North America have emphasised the importance of Gram negative, anaerobic, and legionella organisms as causes of nosocomial respiratory tract infections.

The purpose of the present study was to establish the incidence of lower respiratory tract infections in patients in general surgical wards and to study their pathogenesis and the associated morbidity and mortality.

\section{Methods}

The study was conducted prospectively for six months, starting in November 1987, in four general surgical wards and one urology ward (any "medical" patients overflowing into these wards being excluded). For the purposes of the study, a nosocomial lower respiratory tract infection was defined as "an acute respiratory illness with new respiratory symptoms, occurring more than 48 hours after admission to hospital, which is associated with new focal chest signs on examination and is treated with an antibiotic." We excluded patients having immunosuppressive treatment and those who could be expected to develop such an infection as part of a terminal illness. No patients admitted because of trauma were studied because the City Hospital has no accident and emergency department.

Doctors who diagnosed a nosocomial lower respiratory tract infection sent sputum and blood for culture before antibiotic treatment was started, arranged chest radiography, and informed the study coordinator. Each ward was visited daily by the study coordinator to ensure that additional cases were not missed. On recruitment into the study the patient was interviewed and examined by one of us (WK), and consent for the study was obtained. Further sputum samples and a urine sample were obtained for pneumococcal antigen studies and a throat swab was sent for viral but not bacterial culture. Venous blood was taken for a white blood count; determination of haemoglobin, electrolyte, calcium, $\mathrm{C}$ reactive protein, urea, and creatinine concentrations; liver function tests; serological tests for acute respiratory pathogens; and pneumococcal antigen studies. If clinically indicated, capillary blood was sampled for blood gas estimation.

Repeat venous blood samples were taken for serological tests for respiratory pathogens 10 days after diagnosis if the patient was still in hospital. Patients were examined in an outpatient clinic four to eight weeks after the onset of the respiratory tract infection, when a further blood sample was sent for serological examination for respiratory organisms and a chest radiograph was taken.

\section{Results}

Thirty eight patients were entered into the study, representing $1 \%$ of the total surgical admissions to the wards over the study period. Twelve of the patients had been admitted as an emergency, 22 were male, and the median age was 70 (range 37-90) years. Nineteen were current or recent smokers and eight had 
Table 1 Operative procedures in patients developing postoperative lower respiratory tract infections

\begin{tabular}{lcl}
\hline Operation & $\begin{array}{l}\text { Total No of } \\
\text { operations during } \\
\text { study period }\end{array}$ & $\begin{array}{l}\text { No (\%) developing } \\
\text { lower respiratory } \\
\text { tract infection }\end{array}$ \\
\hline Cystectomy & 4 & $4(100)$ \\
Small bowel resection & 1 & $1(100)$ \\
Repair of perforated ulcer & 2 & $1(50)$ \\
Adrenalectomy & 2 & $1(50)$ \\
Highly selective vagotomy & 2 & $1(50)$ \\
Closure of colostomy & 9 & $2(22)$ \\
Gastrectomy & 15 & $3(20)$ \\
Colectomy & 57 & $8(14)$ \\
Femoral embolectomy & 10 & $1(10)$ \\
Laparotomy & 14 & $1(7)$ \\
Thyroidectomy & 15 & $1(7)$ \\
Cholecystectomy & 98 & $6(6)$ \\
Mastectomy & 100 & $1(1)$ \\
Hernia repair & 228 & $2(1)$ \\
\hline
\end{tabular}

stopped smoking more than six months previously.

Thirty two patients had had an operation during the present admission (table 1) and one had had an upper gastrointestinal endoscopy under intravenous sedation. The remaining five patients were managed without surgery. The median duration of inpatient stay before surgery was two (range 0-21) days. Twenty two patients were operated on through an upper midline incision and six through a lower abdominal incision. None had a subcostal incision. The median duration of the anaesthetic was 95 (range 15-360) minutes. The median time between surgery and development of the lower respiratory tract infection was 2 (range 1-12) days. None of the patients had previously been considered to be at particular risk of developing a respiratory infection, and in no case had specific precautions been taken against the development of nosocomial lower respiratory tract infection beyond those usually taken for patients undergoing such operations.

Sixteen of the 38 patients were receiving antibiotics at the time the infection developed. Three were transferred to the intensive care unit as a result of the lower respiratory tract infection, and two of these were ventilated. Six patients died, including the two patients who were ventilated. Five of the six patients had pre-existing cardiorespiratory disease,

Table 2 Clinical features and results of laboratory investigations

\begin{tabular}{lc}
\hline & No of patients \\
\hline Symptoms & \\
Cough & 35 \\
Sputum & 33 \\
Breathlessness & 8 \\
Chest pain & 1 \\
Pyrexia & 29 \\
Respiratory rate $>20 /$ min & 14 \\
Systolic blood pressure $<100 \mathrm{~mm} \mathrm{Hg}$ & 1 \\
Pulse $>100 /$ min & 10 \\
Lower respiratory tract signs & 38 \\
Radiographic shadowing & 29 \\
White blood count & 23 \\
$>10 \times 10^{9} / 1$ & 1 \\
<4 $\times 10^{9} / 1$ & 14 \\
Sodium $<135$ mmol $/ 1$ & $11^{\star}$ \\
Urea $>7$ mmol $/ 1$ & 8 \\
Liver function tests: $\geqslant 2$ abnormal results & 15 \\
Albumin $<25$ g/1 & $22 \dagger$ \\
C reactive protein $>20$ U/1 & $4 \ddagger$ \\
Arterial oxygen tension $<8 \mathrm{kPa}$ &
\end{tabular}

$\star$ Six patients who had had biliary tract surgery are excluded. + Measured in 24 patients.

$\$$ Measured in 26 patients, two breathing supplementary \$Measured compared with 19 of the 32 survivors ( $p<$ 0.05, Fisher's exact test). Respiratory failure, usually in conjunction with other organ failure, was the cause of death in all six patients, all of whom were over the age of 65 years.

Potential respiratory pathogens were isolated from $21(55 \%)$ of the patients. Sputum from 34 patients was sent for culture. Streptococcus pneumoniae was grown from two patients, Haemophilus influenzae from four, and Staphylococcus aureus from two, one of whom also had Haemophilus influenzae. Pseudomonas spp were grown from the sputum in two cases and "coliform" bacilli in one; in all three cases the patient had received antibiotics postoperatively before developing the infection. Pneumococcal antigen was detected in the sputum from 10 patients, including the two with Strep pneumoniae and the patient with $H$ influenzae in the sputum. Pneumococcal antigen was not detected in any of the urine or serum samples. Antibiotics had been administered before the diagnosis of lower respiratory tract infection in only three of the eight patients with pneumococcal antigen in their sputum in whom Strep pneumoniae was not cultured.

Blood cultures were negative in all 36 of the patients for whom they were performed. Of the 33 throat swabs taken, two grew herpes simplex virus. Paired samples for serological tests for respiratory pathogens were taken from 28 patients, one of whom showed a sixfold rise in Chlamydia psittaci antibody titre and one a fourfold rise in respiratory syncytial virus antibody titre. No change in the mycoplasma or legionella titres was seen in any patient.

The median duration of stay in hospital after operation was 10 (range 1-45) days. This did not differ significantly from the average length of stay for the same operation during the study period. The median duration of antibiotic treatment prescribed for the lower respiratory tract infection was seven (range 122) days. At follow up four to six weeks after discharge nine of 20 repeat chest radiographs remained abnormal, and eight of the $\overline{2} 4$ patients seen still had abnormal physical signs on chest examination.

\section{Discussion}

The incidence of $1 \%$ for nosocomial lower respiratory tract infection in the present study is similar to the incidence in reports from North America. ${ }^{7}$ The importance of risk factors such as abdominal surgery or pre-existing cardiorespiratory disease for the development of postoperative lower respiratory tract infections is well recognised. ${ }^{89} \mathrm{~A}$ causative organism was identified in about half of the patients, again much as in previous studies, ${ }^{10}$ though a higher yield has been obtained with more invasive diagnostic techniques. ${ }^{11}$ The most common pathogen identified was Strep pneumoniae, followed by $H$ influenzae, a pattern similar to that seen with community acquired pneumonia. ${ }^{4}$ These pathogens seem 
likely to have been endogenous and brought into hospital with the patient. Although legionella has been reported as a not uncommon cause of community acquired pneumonia, ${ }^{4}$ we found no cases in this study. Although the use of pneumococcal antigen to determine the aetiology of lower respiratory tract infections is open to criticism, the presence of antigen in the sputum of a patient with pneumonia is a strong indicator that Strep pneumoniae is the causative organism, ${ }^{12}$ and in the British Thoracic Society pneumonia study such patients were accepted as probably having pneumococcal pneumonia. ${ }^{13}$ The failure to grow Strep pneumoniae in patients with pneumococcal antigen in their sputum did not appear to be merely a reflection of prior antibiotic treatment in the present study. Only three cases of possible Gram negative enteric infection were identified, in contrast with studies from North America. The low incidence of Gram negative organisms may reflect the fact that none of the patients had endotracheal tubes in place at the time they developed their respiratory infection, and less than half the patients were receiving antibiotics that could have influenced the oropharyngeal flora. Antibiotics were administered after operation to most of the patients who underwent abdominal surgery, but no conclusions can be drawn from the present study as to whether postoperative prophylactic antibiotics prevent or predispose to lower respiratory tract infection. Provided that nosocomial legionella infection does not occur the antibiotics should be similar to those used for community acquired pneumonia. The routine use of broad spectrum, newer generation cephalosporins or aminoglycosides would seem unnecessary in this setting.

In our hospital there would appear to be few ways in which a change in surgical practice could influence nosocomial pneumonia. The duration of preoperative stay was usually short, routine postoperative antibiotic treatment was not excessive, the surgical teams were aware of the likely pathogens when a patient developed a lower respiratory tract infection, and their choice of antibiotic was appropriate- 29 patients were prescribed an ampicillin derivative and only six received broad spectrum cephalosporins or aminoglycosides. Invasive tests to determine the aetiology of lower respiratory tract infections occurring in general surgical and urological wards would seem to be unnecessary, unless the patient is deteriorating while receiving an apparently appropriate antibiotic. Close surveillance of patients with cardiorespiratory disease in the first few days after an abdominal operation is obviously imperative.

We would like to thank the surgeons of the City Hospital for permission to study their patients, and the staff of the wards included in the study for their cooperation. Simon Andrews kindly provided the data on admission rates and average duration of stay.

1 Santoro J. Nosocomial respiratory tract infections. In: Levison ME, ed. The pneumonias. Boston: John Wright, 1984:183-96.

2 Sanford JP, Pierce AK. Lower respiratory tract infections. In: Bennett JV, Brachman PS, eds. Hospital infections. Boston: Little, Brown and Co, 1979:255-86.

3 Woodhead MA, Macfarlane JT. Is nosocomial pneumonia a problem in Britain? [abstract]. Thorax 1987;42:233.

4 Macfarlane JT, Finch RG. Hospital study of adult community acquired pneumonia. Lancet 1982;ii:255-8.

5 Pugh S. British Thoracic Society pneumonia study: microbiological results [abstract]. Thorax 1985;40:693.

6 Bath JCJL, Boissard GPB, Calder MA, Mofat MAF. Pneumonia in hospital practice in Edinburgh. $\mathrm{Br} J \mathrm{Dis}$

Chest 1964;58:1-16.
7 Wenzel RP, Osterman CA, Hunting KJ. Hospital acquired infections II. Infection rates by site, service and common procedures in a university hospital. Am J Epidemiol 1976;104:645-51.

8 Garibaldi RA, Britt MR, Coleman ML, et al. Risk factors for postoperative pneumonia. Am J Med 1981;70:677-80.

9 Collins CD, Darke CS, Knowelden J. Chest complication after upper abdominal surgery: their anticipation and prevention. Br Med J 1968; i:401-6.

10 White R, Dilworth P. Pneumonia in hospital. Br J Dis Chest 1988;82:121-6.

11 Doron J, Boada J, Falguera $M$, et al. The aetiology of nosocomial pneumonia [abstract]. Bull Eur Physiopathol Respir 1986;22:101S

12 Schmid RE, Anhalt JP, Wold AD, Keys TF, Washington JA. Sputum counterimmunoelectrophoresis in the diagnosis of pneumococcal pneumonia. Am Rev Respir Dis 1979;119:345-8.

13 Research Committee of the British Thoracic Society and the Public Health Laboratory Service. Community acquired pneumonia in adults in British Hospitals 1982-1983. $Q \mathrm{~J}$ Med 1987;62:195-220. 